\title{
New Approach for Automated Detection and Area Calculation of Brain Tumor from MRI Images
}

\author{
Maysaa Abd Ulkareem \\ Department of Computer Information System \\ College of Computer Science and Information \\ Technology \\ University of Basrah
}

\author{
Maha Salih Abdulredha \\ Department of Computer Information System \\ College of Computer Science and Information \\ Technology \\ University of Basrah
}

\begin{abstract}
Medical Image Processing is one of the most challenging and emerging topics in today's research field. Processing of Magnetic Resonance Imaging (MRI) is one of the parts in this field. In recent years, In order to analyze a disease, Physicians consider MR imaging modality is the most efficient one for identification of cancer present in various organs. Therefore, analysis on MR imaging is required for efficient disease diagnosis. This paper describes the proposed strategy to detect and calculating its growth...This proposed method incorporates with some noise removal functions, segmentation and morphological functions which are considered to be the basic concepts of Image Processing. Detection and extraction of tumor from MRI scan images of the brain is done by using MATLAB software
\end{abstract}

\section{General Terms}

Digital image processing

\section{Keywords}

MRI, Brain Tumor, segmentation, morphology, MATLAB.

\section{INTRODUCTION}

A tumor is defined as the abnormal growth of the tissues. Brain tumor is an abnormal mass of tissue in which cells grow and multiply uncontrollably, seemingly unchecked by the mechanisms that control normal cells. Brain tumors can be primary or metastatic, and either malignant or benign [1] .Brain tumors are caused due to abnormal, uncontrolled growth of cells. Primary tumors are those that originate in the brain. Secondary tumors are those that originate in some other part of the body, finally reaching the brain through the process of metastasis. [2]The symptoms of brain tumors include headache, nausea, vomiting, personality and behavioral changes, memory loss, sensory disturbance, weakness, numbness [3], [4] software and determine the complexity of it is known as effort estimation. Incorrect, both underestimates and overestimates of the effort required, complicate scheduling, and management result in over budgets and late in the projects. These reasons represent why effort estimation is somewhat known as sort of critical part [4][5].

\subsection{MRI Scan}

MRI is a fairly new technique that has been used since the beginning of the 1980s. The MRI scanner uses magnetic and radio waves to create pictures of tissues, organs and other structures within the body, which can then be viewed on a computer. There is no exposure to X-rays or any other damaging forms of radiation in MRI

\section{Structure of Brain}

Generally, human brain includes three major parts controls different activity.

\subsection{Cerebrum}

The cerebrum controls learning, thinking, emotions, speech, problem solving, reading and writing. It is divided into right and left cerebral hemispheres. Muscles of left side of the body control by right cerebral hemispheres and muscles of right side of the body control by left cerebral hemispheres[5].

\subsection{Cerebellum}

The cerebellum controls movement, standing, balance and complex actions.

\subsection{Brain stem}

Brain stem joints the brain with spinal cord. Brain stem controls blood pressure, body temperature and breathing and controls some basic functions [6].

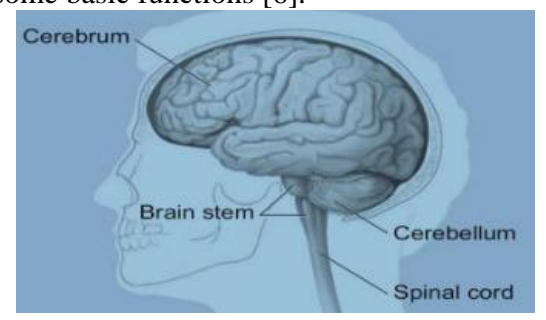

Fig1: Indicate the brain structure [6]

MR image provide details information about human anatomical structure and tissues. Also MR image is safe compare to CT scan and X- Ray Image. It is not affect the human body. MR Image is providing information for use of further treatment and research area. Fig.2 shows the brain MRI image with the information about different tissues [7].

A Study of Segmentation Methods for Detection of Tumor in Brain MRI

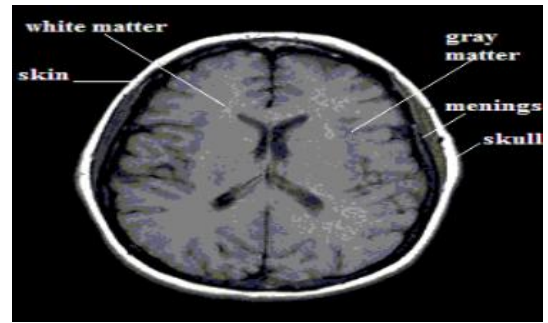

Fig 2: Brain MR Image [8] 


\section{CONVENTIONAL METHOD}

Magnetic resonance imaging (MRI) segmentation is a complex issue. This paper proposes a new method for estimating the right number of segments and automatic segmentation of human normal and abnormal MR brain images. A wide variety of segmentation methods has been proposed. However, there is no standard approach which yields successful results for MRI brain segmentation or clinically acceptable. In general segmentation techniques are divided into four categories.

\section{PROPOSED WORK}

The proposed work used basic concepts to detect tumor in this paper, the component of the image hold the tumor generally has extra concentration then the other segment and guess the area, We calculate the area in pixel.

\subsection{ALGORITHM FOR DETECTING BRAIN TUMOUR}

Input: MRI of brain image.

Output: Tumors portion of the image.

Step 1:- Read the input RGB image.

Step 2:- Resize this image in to $200 \times 200$ image matrix.

Step 3 Convert the input image from RGB color space to HSV color space

Step 4:- Now we can enhance our image by duplicate the value of 2 nd and 3 rd diminution of HSV image.

Step 5:- Computes a global threshold that can be used to convert an intensity image (Step 4) to a binary image with a normalized intensity value which lies in between range 0 and 1.

Step 6:- Compute the morphological operation by two mat lab command imopenandsteel with arbitrary shape.

Step 7:- Store the size of the step 6 image into var1 and var2 \begin{tabular}{l} 
i.e. no. Of rows and column in pixels by: [var1 \\
\hline
\end{tabular} var2] $=\operatorname{size}($ step 6 image)

Step 8:- For $\mathrm{i}=1: 1$ :var1

Step 9:- For $\mathrm{j}=1: 1$ :var2

Step 10:- If step 6 image $(\mathrm{i}, \mathrm{j})==1$

Step 11:- step 1 image $(\mathrm{i}, \mathrm{j})=255$

Step 12:- Else

Step 13:- step 1 image $(\mathrm{i}, \mathrm{j})=$ step 1 image $(\mathrm{i}, \mathrm{j}) * 0.5$

Step 14:- End

Step 15:- End

Step 16:- End

Step 17:- Convert in to binary image and traces the exterior boundaries of objects, as well as boundaries of holes inside these objects, in the binary image and into an RGB color image for the purpose of visualizing labeled regions.

\subsection{ALGORITHM FOR AREA CALCULATION \\ Input: Tumor portion of the image.}

Output: Area of the tumor.

Step 1:- Read the input gray scale image.

Step 2:- Resize this image in to $200 \times 200$ image matrix.

Step3:- Compute numbers of rows and column in pixels by: $[\mathrm{r} 2 \mathrm{c} 2]=\operatorname{size}(\mathrm{J})$

Step 4:- Initialize a variable $\mathrm{a}=0$

Step 5:- For $\mathrm{i}=1: 1: \mathrm{r} 2$

Step 6:- For $\mathrm{j}=1: 1: \mathrm{c} 2$

Step 7:- If I $(\mathrm{i}, \mathrm{j})==255$

Step 8:- $\mathrm{a}=\mathrm{a}+1$

Step 9:- Else

Step 10:- $\mathrm{a}=\mathrm{a}+0$

Step 11:- End for

Step 12:- End for

Step 13:- End if

Step 14:- Display the area a.

\section{RESULTS AND DISCUSSION}

\subsection{Detection of Brain Tumor}

Using MATLAB program we got the following images as results in brain tumor detection


3rd \& 4th step
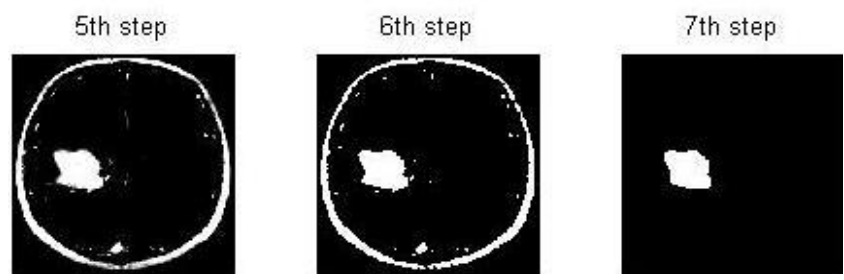

\subsection{AREA CALCULATION OF TUMOUR REGION}

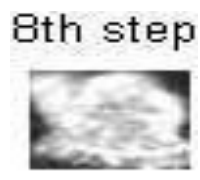

Area of above detected brain tumor 1179 


\subsection{Some other Results are shown below}
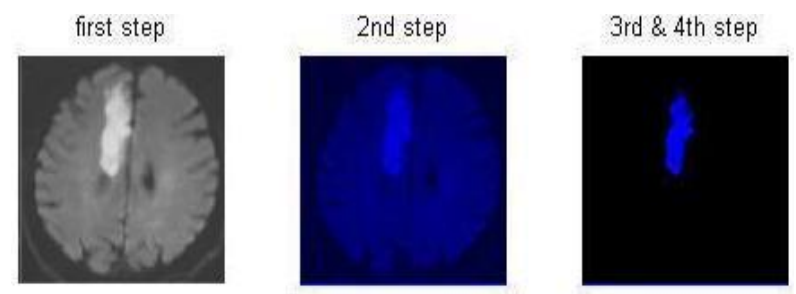

5th step

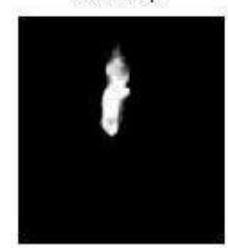

6th step
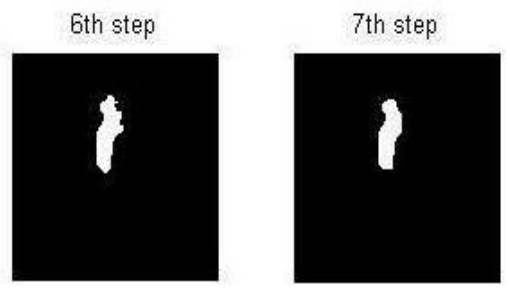

Bth step

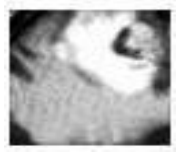

Area of above detected brain tumor 2311
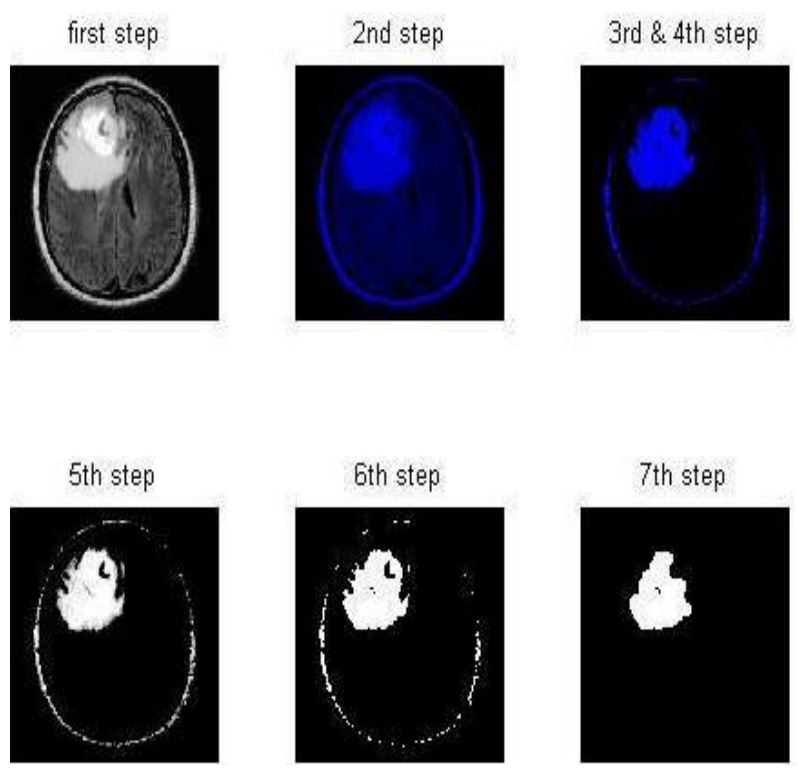

\section{8th step}

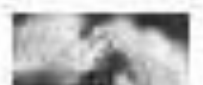

Area of above detected brain tumor 272

\section{COMPARATIVE ANALYSIS}

The comparative analysis is presented in Table 1 :
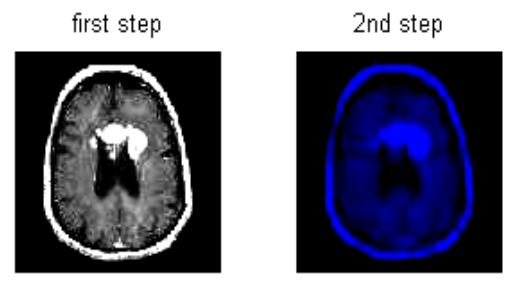

3rd \& 4th step

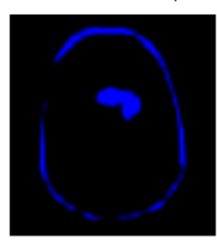

5 th step

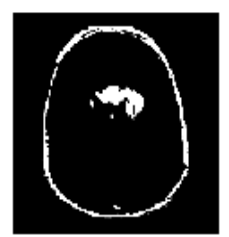

6th step

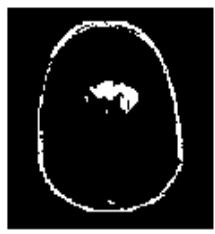

7 th step

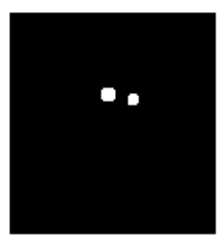

Bth step



Area of above detected brain tumor 950
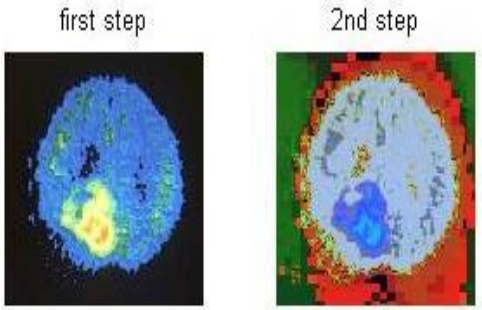

3rd \& 4th step

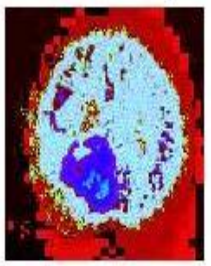

5 th step
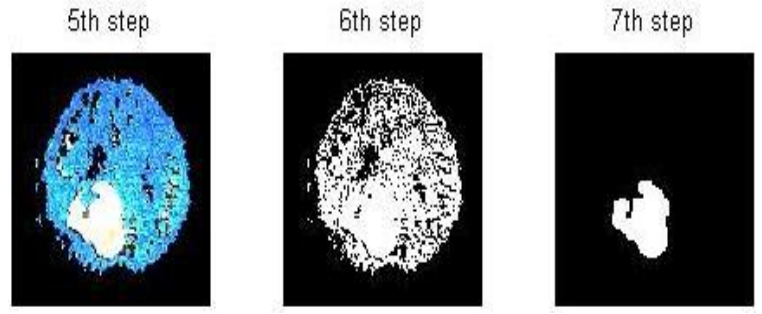

\section{Bth step}

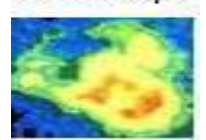

Area of above detected brain tumor 2240 


\begin{tabular}{|c|c|c|c|}
\hline Image name & Image size & Size of tumor & Execution time \\
\hline Img1 & $220 \times 250$ & 2311 & 0.154 \\
\hline Img2 & $676 \times 624$ & 1179 & 0.129 \\
\hline Img3 & $233 \times 287$ & 950 & 0.092 \\
\hline Img4 & $200 \times 200$ & 272 & 0.051 \\
\hline Img5 & $200 \times 219$ & 2240 & 0.172 \\
\hline
\end{tabular}

\section{CONCLUSION}

MRI images are best suitable for brain tumor detection. In this study Digital Image Processing Techniques are important for brain tumor detection by MRI images. The preprocessing techniques include different methods like Filtering, Contrast enhancement, Edge detection is used for image smoothing. The preprocessed images are used for post processing operations like; threshold, segmentation and morphological, which is used to enhance the images.. In this paper, the extraction of the tumor and the size of the interested tumors successfully obtained through a few steps in the MATLAB coding for image processing. We were also able to segment the different part of the brain from the brain CT mages. By calculating the area, we can know that there are slight differences between the sizes of the tumor for different slice of brain images.

\section{FUTURE WORKS}

The following works are suggested to be future works :

1. Using Decision tree classification technique tumor has been found as well as classified in Normal or Abnormal class.

2. The proposed system can be extended for some other modality of images like CT, Ultra Sound etc., for different organs of human such as Breast, Brain and so on.

3. Can use A fuzzy clustering based segmentation of any brain MRI image has also been provided which can be used to Study the intricate internal brain structure.

\section{REFERENCES}

[1] Mohamed Lamine Toure, 2010 ."Advanced Algorithm for Brain Segmentation using Fuzzy to Localize Cancer and Epilepsy Region", International Conference on Electronics and Information Engineering (ICEIE 2010), Vol. no 2.

[2] Neha Tirpude \& Rashmi Welekar, 2013 “Automated Detection and Extraction of Brain Tumor from MRI Images", International Journal of Computer Applications (0975 - 8887) Volume 77- No.4,( September 2013).

[3] D.A. Dahab, S.A. Ghoniemy , G.M. Selim, 2012. "Automated Brain Tumor Detection and Identification using Image Processing and Probabilistic Neural Network techniques". International Journal of Image Processing and Visual Communication, 1-8 .

[4] Jay Patel1 and Kaushal Doshi2 .2014 "A Study of Segmentation Methods for Detection of Tumor in Brain MRI", Advance in Electronic and Electric Engineering .ISSN 2231-1297, Volume 4, Number 3 (2014), pp. 279284, (ResearchIndia .

[5] J.Viner.2013, "Brain Tumors. University of California, San Francisco Department of Neurosurgery", Availableathttp://nursing.ucsf medicalcenter.org/education/class Material/34_2.pdf.

[6] National cancer Institute, "What you need to know about Brain tumors".

[7] [http://www.braintumor.org]

[8] Segmentation Techniques for Brain Images, International Journal of Computer Technology and Electronics Engineering (IJCTEE), Vol.3, Special Issue( MarchApril-2013 